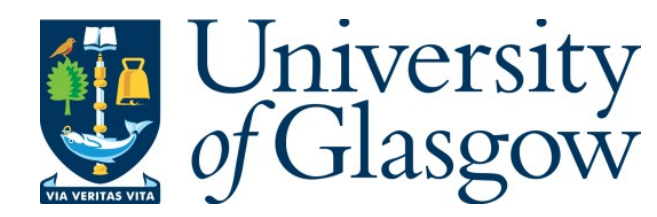

Deng, L., Bradshaw, A. C., and Baker, A. H. (2016) Role of noncoding RNA in vascular remodelling. Current Opinion in Lipidology, 27(5), pp. 439-448.

There may be differences between this version and the published version. You are advised to consult the publisher's version if you wish to cite from it.

http://eprints.gla.ac.uk/122352/

Deposited on: 22 September 2016

Enlighten - Research publications by members of the University of Glasgow http://eprints.gla.ac.uk 


\title{
Role of Noncoding RNA in Vascular Remodelling
}

\author{
Lin Deng ${ }^{1,2}$, Angela Bradshaw ${ }^{1}$, Andrew H Baker ${ }^{2}$ \\ *Correspondence: Andrew H Baker, Centre for Cardiovascular Science, University of Edinburgh, \\ Queen’s Medical Research Institute, 47 Little France Crescent, Edinburgh UK, EH16 4TJ, Tel: 0131 \\ 242 6728, Email: Andy.Baker@ed.ac.uk \\ ${ }^{1}$ BHF Glasgow Cardiovascular Research Centre, University of Glasgow, Glasgow, UK. \\ ${ }^{2}$ BHF/University of Edinburgh Centre for Cardiovascular Science, Edinburgh, UK
}

Purpose of review: Non-coding RNA (ncRNAs), such as microRNAs (miRNAs) and long non-coding RNAs (LncRNAs), are becoming fundamentally important in the pathophysiology relating to injury-induced vascular remodelling. We highlight recent studies that demonstrate the involvement of ncRNAs in vein graft disease, in in-stent restenosis (ISR) and in pulmonary arterial hypertension (PAH), with a particular focus on endothelial cell (EC) and vascular smooth muscle cell (VSMC) function. We also briefly discuss the emerging role of exosomal-derived ncRNAs and how this mechanism impacts on vascular function.

Recent findings: NcRNAs have been described as novel regulators in the pathophysiology of vascular injury, inflammation and vessel wall remodelling. In particular, several studies have demonstrated that manipulation of miRNAs can reduce the burden of pathological vascular remodelling. Such studies have also shown that exosomal miRNA-mediated, cell-to-cell communication between ECs and VSMCs is critical in the disease process. In addition to miRNAs, lncRNAs are emerging as regulators of vascular function in health and disease. Although lncRNAs 
are complex in both their sheer numbers and mechanisms of action, identifying their contribution to vascular pathology is essential.

Summary: Given the important roles of ncRNAs in vascular injury and remodelling together will their capacity for cell-to-cell communication, manipulating ncRNA might provide novel therapeutic interventions.

Keywords: Non-coding RNA, Vascular remodelling, in-stent restenosis, vein graft, pulmonary arterial hypertension

\section{Introduction}

Vascular remodelling after acute injury is a multifactorial pathological process that contributes to cardiovascular disease, including ISR, PAH, atherosclerosis, and others $[1,2]$. The remodelling process involves structural changes of the vessel as a consequence of altered cell proliferation/growth, cell apoptosis/death, cell migration, and modulation of the production and degradation of extracellular matrix (ECM) [3, 4]. Both endothelial and smooth muscle cells are key mediators of the pathological response to acute vascular injury, driving the progressive remodelling process that characterizes neointimal formation [5, 6]. In addition, inflammatory activation of the vessel wall also contributes to vascular remodelling [7]. Endothelial injury, such as the endothelial denudation that arises after implantation of stents into coronary vessels, is an important early contributor to the initiation of vascular inflammation [8]. Following injury, growth factors and cytokines released from ECs and inflammatory cells promote smooth muscle cell proliferation, migration and ECM protein deposition [9, 10]. Although multiple studies over the last two decades have focused 
on delineating the molecular mechanisms that drive the remodelling response to vascular injury [11-13], with the exemption of drug-eluting stents, there is currently a paucity in effective therapeutics, necessitating further research and innovative thinking around molecular mechanisms that might govern pathological responses

According to recent transcriptome analyses, protein-coding genes actually account for only $1.5 \%-2.0 \%$ of the human transcriptome. The transcriptional output of the larger portion of the transcriptome (i.e. 98\%) consists of non-coding RNA species, including miRNAs (<200nt) and LncRNAs >200nt [14]. MiRNAs have been intensively studied for the last decade [15, 16], However, IncRNA structure and function is an emerging field in the vascular arena, with the publication of several studies recently that suggest an important contribution to pathological vascular remodelling processes.

\section{MiRNA biogenesis and function}

MiRNAs are a novel class of endogenous, small/short non-coding transcripts of 16 to 29 nucleotides in length RNA that negatively regulate gene expression via degradation or translational inhibition of their target mRNAs. The biogenesis of miRNA is well reviewed in several highly cited publications [17-19]. MiRNAs are regarded as essential regulators in physiology and pathophysiology of the cardiovascular system $[20,21]$. They are becoming an intriguing target for therapeutic intervention for a number of reasons. First, a single miRNA can regulate several hundred target genes (including targets in the same or complimentary cellular 
pathways) making miRNAs potentially very powerful disease modifiers [22]. Second, several lines of evidence demonstrate that miRNAs are essential regulators of VSMC development, differentiation, and contractile function offering a rich repertoire of disease-modifying therapeutics [23]. Finally, RNA therapeutics benefit from a great deal of prior translational research (e.g. SiRNA and exon skipping technology) that suggest RNA therapeutics might be attractive, albeit with specific challenges such as delivery, off target effects etc. [24].

\section{The role of miRNAs in pathological vascular remodelling}

Neointimal formation is a hallmark of many vascular diseases. VSMCs are one of the major cellular components of the vessel wall and are implicitly involved in neointimal formation [25]. For example (and aligned to our own research) in vein graft disease, VSMC proliferation and migration contributes to the increased vein graft medial thickening, neointimal formation and superimposed atherosclerosis [26]. Similarly, the proliferation of VSMC in response to vascular injury following angioplasty and stent implantation leads to neointimal formation and restenosis [27]. Further, a key feature of PAH is pulmonary vascular remodelling and development of occlusive and plexiform intimal lesions in the small, distal pulmonary vessels [28]. In this section, we discuss recent findings on the regulation of pathological vascular remodelling by miRNAs.

MiR-21 first identified to be significantly increased in rat carotid arteries following balloon injury [29]. In VSMCs, knockdown of miR-21 inhibited cell proliferation, decreased migration and induced apoptosis by targeting important 
regulators of cellular function, including PTEN and Bcl-2 [29], PDCD4 and activator protein 1 [30], tropomyosin 1 [31], while overexpression of miR-21 induced human aortic SMC proliferation and reduced VSMC differentiation by targeting specificity protein-1 (SP1) [32]. Landmark studies also showed that miR-21 is involved in the regulation of TGF- $\beta$ and BMP signalling, activation of which led to increased expression of miR-21 via post-transcriptional mechanisms [33, 34]. These signalling pathways are central mediators of the vascular remodelling process following acute injury, regulating VSMC proliferation, migration, and phenotype [35]. Similar to the Ji et al study described above, upregulation of miR-21 has been observed across many in vivo models of vascular injury, including mouse and pig models of vein graft failure, and mouse models of ISR [29] [36] [37**] [38**]. These studies also underlined the therapeutic potential of targeting this broadly-expressed miRNA, showing that pharmacological inhibition of miR-21 could decrease neointimal formation in injured rat carotid arteries following angioplasty, while anti-miR-21 coated stents effectively reduced ISR by inhibiting VSMC proliferation following implantation [29] [38**]. Consistent with these findings, genetic ablation of miR-21 significantly reduced neointimal formation in a mouse vein graft failure model[36] and blocked neointimal formation in a mouse model of ISR by reducing VSMC proliferation and migration, macrophage polarization, and inflammatory activation[37**]. Mechanistic studies have identified several target genes of miR-21, suggesting complex mechanism of action dictated by both expression across a range of cell types and targeting transcriptome within those cells in a dynamic, 
pathologically regulated manner [36-38**]. In addition, the expression of miR-21 in plasma samples from patients with ISR following implantation of drug-eluting stents were significantly higher than in non-ISR patients and healthy controls, which showed that miR-21 could act as a potential biomarker for the development of ISR [39]. In PAH, manipulation of miR-21 levels has also been shown to affect the vascular remodelling that leads to the formation of obstructive lesions in the pulmonary vasculature. Sequestration of miR-21 by antimiR-21 treatment diminished chronic-hypoxia-induced pulmonary hypertension $(\mathrm{PH})$ and attenuated pulmonary vascular remodelling [40]. However, miR-21 knockout mice displayed exaggerated manifestations of PH in SU5416 and chronic hypoxia models [41*, 42]. This data, while conflicting, highlights the complex role that miR-21 might play in vascular remodelling in the distal vasculature in the lung. Further studies are now required to identify the mechanisms underlying these divergent phenotypes, potentially by exposing miR-21 conditional knockout animals to different experimental PAH induction protocols, or by utilising novel strategies to inhibit miR-21 in situ. The role of miR-21 in PAH has been extensively reviewed elsewhere [43]. In a translational context, manipulation of miR-21 in different pre-clinical disease models has shown positive therapeutic effects, although it would be important to develop strategies for localised targeting of the miR-21 inhibitors to the vessel wall, such as in the case of eluting stents. Interestingly, a chemically modified oligonucleotide antimiR-21 manufactured by Regulus Therapeutics (San Diego, CA92121, United States) has entered phase 1 clinical trials for the treatment of Alport Syndrome, a 
disease of the glomerular basement membrane, where miR-21 inhibition is aimed at targeting abnormal renal function [44].

In addition to miR-21, several other miRNAs have been shown to play critical roles in the pathological response to acute vascular injury. MiRNAs have been shown to mediate the effects of many known regulators of vascular disease, such as PDGF, TGF- $\beta /$ BMP, and the interleukin family of inflammatory cytokines. For example, miR-146b-5p is necessary for PDGF-induced VSMC phenotype transition [45]. MiRNAs that control vascular functions by affecting TGF- $\beta$ family signalling have been reviewed elsewhere [46]. MiRNAs also play a central role in the modulation of vascular cell behaviour and function during disease pathogenesis, such as Let-7a and miR-221in vein graft disease [47] [48], the miR-143/145 cluster in ISR and PAH [49] [39] [50**] and miR-30c [51], miR-322 [52], miR-130a [53] in PAH. An overview of confirmed target genes for these miRNAs are showed in Table 1.

In summary, many miRNAs are now known to be associated with the development of vascular remodelling processes in vivo and in vitro, while manipulation of several miRNAs have shown therapeutic efficacy in animal models of vascular disease. However, many facets of miR biology remain to be fully elucidated, including their precise mechanisms of action, cell selectivity in effect and the factors underlying their diverse effects. Moreover, as previously stated, there is currently a lack of effective pharmacological strategies for the direct targeting of miRNA in disease pathogenesis. Thus, much works remains to be done to fully understand, target and therapeutically manipulate the biological roles of miRNAs in 
vascular disease.

\section{LncRNA transcription and function}

LncRNAs, in common with mRNAs, are generally transcribed by RNA Polymerase II, and further spliced, 5'-capped (m7G), and 3'-polyadenylated [54]. The transcriptional regulation of lncRNA is well reviewed elsewhere [55]. LncRNA are, in general, expressed at relatively low levels and are far less well conserved than miRNA [56-58]. LncRNA can be classified into different subtypes based on their genomic location: sense lncRNAs, antisense lncRNAs, bidirectional lncRNAs, intronic lncRNAs and intergenic lncRNAs [59]. LncRNAs have diverse molecular mechanisms of regulating gene expression through interactions with DNA, RNA, or protein, by acting as decoys, scaffolds, guides or signalling molecules (Figure 1). In addition, lncRNAs can regulate gene expression post-transcriptionally, for example by modulating splicing, mRNA translation and mRNA degradation, or by acting as miRNA sponges. There are several comprehensive reviews summarized the functions and mechanisms of lncRNA [55, 60-63].

\section{Role of IncRNAs in vascular remodelling}

Accumulating early evidence suggests that lncRNAs may play an important role in vascular pathophysiology. Early studies identified antisense noncoding RNA in the INK4 locus (ANRIL), a lncRNA expressed in VSMC, which was able to regulate CDKN2A/B expression in aortic VSMC and attenuate proliferation in atherosclerosis [64-66] as well as modulate inflammatory responses in coronary artery disease [67]. 
Lnc-Ang362, which hosts the miR-221 and miR-222 genes, was shown to be regulated by angiotensin II in VSMC, and was in turn able to modulate VSMC proliferation, vascular remodelling and neointimal hyperplasia in concert with miR-221 and miR-222 [68-70]. These studies showed that lncRNA were involved in the control of cell proliferation, and could be regulated alongside proximal miRNAs in VSMCs. RNA-Seq analysis of human coronary artery SMC revealed a novel lncRNA, Smooth muscle and Endothelial cell-enriched migration/differentiation-associated long NonCoding RNA (SENCR), which is expressed in both VSMCs and ECs In VSMCs, knockdown of SENCR decreased the expression of myocardin and numerous smooth muscle contractile genes and increased a number of pro-migratory genes (namely Midkine (MDK) and Pleiotrophin (PTN)). Inhibition of these two genes reversed the increased VSMC migration mediated by SENCR silencing, which suggested that the expression of these genes was partially regulated by SENCR in these cells. However, the functions of SENCR in endothelial cells were not reported in this study [71*]. Our group further revealed that SENCR induced the proliferation, migration and angiogenesis of Human Umbilical Vein Endothelial Cells (HUVECs). In patient samples, SENCR expression was altered in vascular tissues and cells derived from patients with critical limb ischemia and premature coronary artery disease compared with healthy controls [72]. These two studies revealed that SENCR could modulate the behaviour of both SMC and EC, and exhibited altered expression patterns in vascular tissues and cells from vascular disease patients. However, the exact molecular targets and mechanisms of 
SENCR in pathological vascular remodelling remain to be determined.

Similar to SENCR, LincRNA-p21 is expressed in both VSMC and EC. In human VSMCs, lincRNA-p21 repressed cell proliferation and induce apoptosis in vitro. LincRNA-p21 also inhibited neointimal formation in carotid arteries and repressed proliferation and induced apoptosis in carotid artery injury model in vivo. The authors further confirmed the expression level of lincRNA-p21 was decreased in patients with coronary heart disease [73**]. In mouse EC, lincRNA-p21 induced cell apoptosis and cell cycle progression. In addition, lincRNA-p21 inhibited cell proliferation through binding miR-130b [74]. Unlike many lncRNAs, LincRNA-p21 contains a very highly conserved 780 nt length 5' region in mammals, which provided the possibility of using an in vivo animal model to study the role of this lncRNA [75]. These data identified lincRNA-p21 is a key regulator of cell proliferation and apoptosis in vascular cells, which are primary contributors to vascular remodelling. Consistent with the human in vitro data, knockdown of lincRNA-p21 resulted in dramatic neointimal hyperplasia in a mouse model of carotid artery injury, which indicated that lincRNA-p21 could serve as therapeutic target to treat vascular remodelling diseases [73**].

Using RNA-Seq, we recently defined a novel smooth muscle specific lncRNA SMILR, which could be induced by IL-1 $\alpha$ and PDGF in human saphenous vein smooth muscle cells (HSVSMCs). Knockdown of SMILR markedly reduced HSVSMC proliferation, likely through regulation of the HAS2 gene, located proximal to the genetic locus for SMILR. In human samples, SMILR expression increased in 
unstable atherosclerotic plaques and plasma from patients with high plasma C-reactive protein [76**]. These data suggested that inhibiting SMILR expression could be a potential target for treating vascular diseases characterised by VSMC proliferation.

In the context of EC, studies have shown that the lncRNA Metastasis associated lung adenocarcinoma transcript 1 (MALAT1) could regulate retinal vessel remodelling by affecting retinal EC proliferation, migration and tube formation, as genetic ablation of MALAT1 inhibited the proliferation of EC and reduced neonatal retina vascularization in vivo $\left[77,78^{* *}\right]$. In human aortic EC, knockdown of the lncRNA H19 reduced cell proliferation and activated p21/CDKN1A, increasing apoptosis, decreasing angiogenesis, and diminishing the number of cells in G0/G1 and S phases in cell cycle [79]. These two lncRNAs that are highly expressed in EC showed multifunctional features both in vivo and in vitro. EC lncRNAs have been also reviewed elsewhere [80-82]. An overview of target genes/miRNAs of these lncRNAs is given in Table 1.

In summary, there are few lncRNAs that have been studied in depth in the context of vascular disease, with most studies simply reporting the dysregulation of vascular cell function after manipulation of the lncRNAs. The direct targets and mechanisms of many lncRNA remain largely unknown, so urgent attention is needed in this context, particular relating to their mechanism of action. Unfortunately, the low conservation of many lncRNAs restricts the availability of in vivo disease models, which is a significant impediment to translation of lncRNA therapeutics to the clinical 
arena. Therefore, much more work is required to fully explore the role of lncRNAs in the pathophysiological processes driving vascular disease, sometimes using innovative models with human cells and tissues.

\section{Vascular cell-to-cell communication through miRNAs}

Cell-to-cell communications can be mediated by extracellular vesicles, exosomes, apoptotic bodies, microparticles, and tunnelling nanotubes (TNTs) [83, 84]. Exosomes are 40 to $100 \mathrm{~nm}$ in size and represent a specific subtype of secreted membrane vesicles formed through the fusion of multivesicular endosomes with the plasma membrane [85]. Exosomes are released by many types of cell, and have been identified in most body fluids, including plasma, urine, saliva, cerebrospinal, amniotic and synovial fluids. Exosomes are now known to carry a wide array of molecules including: proteins, DNAs, mRNAs, miRNAs and lncRNAs, depending on a variety of factors including the cell type from which the exosome originates, the state of health of the host, and extracellular stimuli [86]. Exosomes can be taken up by neighbouring or distant cells and can be functional in the recipient cells by fusion with the plasma membrane, via receptor-mediated uptake or by internalisation via endocytosis or macropinocytosis [87]. Since the discovery of miRNA in exosomes, a number of studies have focused on the identification and function of exosomal miRNAs in cancer [88] and cardiovascular diseases [89-93]. Interestingly, cancer cell derived exosomes contain key components of miRNA biogenesis including the proteins RNA-induced Silencing Complex (RISC), Argonaute-2 (AGO2), Dicer, and 
TAR RNA-binding protein (TRBP), which displayed cell-independent capacity to process precursor miRNAs into mature miRNAs [94]. Here we focus on the role of exosomal miRNAs in vascular cells.

An early study showing the importance of exosomal miRNAs in vascular cells evaluated exosomal miR-143/145 in atherosclerosis. This study showed that miR-143/145 can affect vascular cell function following exosomal transport, via cell-to-cell communication. Kruppel like factor 2 (KLF2) or shear-stimulated HUVECs secreted miR-143/145 enriched exosomes, which could be taken up by VSMCs to derepress the targets genes of miR-143/145 (such as EST domain-containing protein Elk-1 (ELK1), Krupple like factor 4 (KLF4), Matrix metallopeptidase 3 (MMP3), Slingshot protein phosphtase 2 (SSH2)) and induce an atheroprotective SMC phenotype both in vitro and in vivo [95]. In the pulmonary vasculature, we recently found that exosomal miR-143 derived from pulmonary artery smooth muscle cells (PASMCs) can be transferred to pulmonary artery endothelial cells (PAECs) exerting pro-migratory and pro-angiogenic effects [50**]. Apart from exosome-mediated transfer, TGF- $\beta$ triggered miR-143/145 transfer to ECs from VSMCs via TNTs. Over-expression of miR-143/145 in ECs had anti-angiogenic effects and reduced EC proliferation by directly targeting hexokinase 2 (HKII) and Integrin beta-8 (ITG $\beta 8$ ) separately [96**]. These data demonstrated the vital role of miR-143/145 in vascular cell proliferation, migration, and angiogenesis. Other EC secreted miRNAs including miR-126 that could be taken by VSMCs via Ago2-facilitated miRNA transmission [97], miR-26a [98*] and miR-214 [99]. The 
cell-to-cell communication between vascular cells by miRNAs is summarised in Figure 2. This is a relatively new field and there is much to discover about miRNA regulation and function through secretion and cell: cell communication.

Exosomal lncRNAs have been found in several cancer cells and patient plasma. These include lncRNA HOX transcript antisense RNA (HOTAIR) [100], H19 [101], Long intergenic Non-coding RNA, Regulator Of Reprogramming (lncRNA ROR) [102], Linc-very-low-density lipoprotein receptor (Linc-VLDLR) [103], lncRNA transcript UC.339 (lncRNA TUC339) [104], lncRNA-p21 [105] and other lncRNAs [106]. However, there is no report about exosomal lncRNAs secreted by the vascular cells. Recently, our group demonstrated that smooth muscle enriched lncRNA Smooth muscle enriched long noncoding RNA (SMILR) was secreted into the cell culture media but was located in a non-exosomal or microparticle fraction [76**]. More studies should be performed to investigate the secretory pathways utilised by this IncRNA, the function of the secreted lncRNA molecules and the compartment or fraction that the lncRNA is located within. More generally, it would be important to identify whether cell-cell communication mediated by exosomal or microparticle lncRNAs plays a role in regulating the behaviour of vascular cells during vascular remodelling processes.

\section{Therapeutic prospective of ncRNAs}

The translation of ncRNAs from bench to bedside has received considerable attention in recent years. In general, the therapeutic approaches involve the inhibition or 
overexpression of ncRNAs. Several approaches to manipulate ncRNAs are currently in use pre-clinically, including miRNA antagonists, miRNA mimics, antisense oligonucleotide (ASO), and small interfering RNA (siRNA). The first anti-miRNA (Miravirsen) to enter clinical trials is a locked nucleic acid-modified DNA phosphorothioate antisense oligonucleotide targeting miR-122, which sequesters mature miR-122 to treat patients with hepatitis C. Miravirsen is currently in phase 2 clinical trials [107, 108]. MiR-RX34 (MRX34), a double-stranded miR-34 RNA mimic encapsulated in a liposomal nanoparticle formulation, is the first miRNA mimic to enter phase I clinical trials [109]. Several antimiRNA/mimic therapeutics targeting miRNAs are currently under development, including Let-7, miR-21, miR-208, miR-195, miR-221, miR-29, miR-155, miR-10b, and miR-103/107. Four of these miRNA-based therapeutic drugs have entered into phase 1 clinical trials [110-114*]. Despite intensive studies of miRNAs in vascular disease, there is no miRNA-based therapeutics entering clinical trials at present in this area. One of the obstacles impeding development of miRNA-based therapeutics is low delivery efficacy to the vasculature and the likely need for repeated delivery. There are several strategies to deal with miRNA-based therapeutic challenges, which are summarised and discussed in this review [114*]. However, despite the obstacles, pharmacological manipulation of disease-associated miRNAs shows promising therapeutic potential in animal disease models. With more and intensive studies of miRNAs in vitro and in vivo pre-clinical models and human patients, the application of miRNA-based therapeutics in the clinic can become a reality. For lncRNAs, there are also several 
obstacles facing the development of lncRNA-based therapeutics. First, the functions and mechanisms used by lncRNAs are much more complex and diversified than miRNAs [115]. In spite of recent studies, lncRNAs are still largely an "unknown" with regard to their cellular functions and molecular mechanisms. Second, the majority of lncRNAs that localise to the nucleus are thought to act as epigenetic regulators [116]. This feature makes it hard to target lncRNAs using siRNA, which is a potential therapeutic strategy. Third, most lncRNAs lack conservation between species, which restricts the utility of pre-clinical disease models. One possible strategy to overcome these issues is to identify the direct targets of disease-associated lncRNAs and use pre-clinical disease models to assess the therapeutic role of these targets in disease pathogenesis and/or use innovative human-based model systems $e x$ vivo and in vitro.

\section{Conclusions}

Recent studies have dramatically improved our understanding of the multifaceted roles that ncRNAs play in pathological vascular remodelling. The expression of several miRNAs have been shown to be dysregulated in various animal disease models as well as in patient samples, while novel direct targets of these disease-associated miRNAs have been validated. Manipulation of these miRNAs in pre-clinical animal models has showed therapeutic benefit. However, there are still some less-understood aspects of miRNA biology that require further study, for example the role(s) of miRNA passenger strands (which in some cases have been 
shown to be functional), in vivo targeting (as most targeted studies of miRNAs are carried out in vitro) and miRNA: miRNA or miRNA: lncRNA interactions. Compared with miRNAs, only few LncRNAs have been investigated in depth in vascular disease. The cellular function and biological mechanisms utilised by these lncRNAs still requires further exploration. Taken together, the evidence summarised in this review reveals that targeting ncRNAs may be a promising therapeutic strategy for the treatment of diseases characterised by vascular remodelling.

\section{Key points}

- $\quad$ Noncoding RNAs have emerged as critical regulators of diseases characterised by pathological vascular remodelling.

- $\quad$ MiRNAs have been shown to regulate VSMC proliferation, migration, apoptosis and phenotype switching.

- Accumulating studies indicate that lncRNAs may critically regulate vascular pathophysiology. Deep sequencing will identify more lncRNAs involved in the regulation of vascular cell behaviour and disease pathogenesis.

- Exosomal miRNAs from vascular cells play an important role during the development of atherosclerosis and PAH by regulating SMC and EC function.

- $\quad$ Based on extensive studies in pre-clinical models of vascular disease and in patient samples, several clinical trials using various approaches to manipulate ncRNA (e.g. anti-miRNAs, miR mimics) are now in progress.

\section{Acknowledgements}


None

\section{Financial support and sponsorship}

This review is supported by a Chair grant from British Heart Foundation to AHB, a Royal Society of Edinburgh (RSE) Personal Research Fellowship to ACB, and a China Scholarship Council PhD Studentship (to LD; 201206240013).

\section{Conflicts of interest}

None

Table 1 Overview of confirmed target genes of ncRNAs in VSMCs

\begin{tabular}{|c|c|c|}
\hline ncRNA & Target genes & Effect on cell behaviour/neointimal formation \\
\hline \multirow[t]{8}{*}{ miR-21 } & $\operatorname{PTEN}^{\left[29,38^{* *}\right]}, \mathrm{BCL} 2^{[29]}$ & VSMC proliferation and apoptosis \\
\hline & $\operatorname{PDCD} 4^{\left[30,37^{* *}\right]}$ & VSMC apoptosis, proliferation, and migration \\
\hline & $\mathrm{TPM}^{[31]}$ & VSMC proliferation and migration \\
\hline & $\mathrm{SP} 1^{[32]}$ & VSMC proliferation and differentiation \\
\hline & DOCK $4,5,7^{[35]}$ & VSMC migration and contractility \\
\hline & STAT3 ${ }^{[36,37 * *]}$, PTEN, & \\
\hline & $\mathrm{BMPR}^{[36]}$ & VSMC proliferation, migration; modulates neointimal formation \\
\hline & BMPR2, STAB1, YOD1 ${ }^{[40]}$ & PASMC proliferation and apoptosis \\
\hline miR-221 & $\mathrm{CDKN}_{1} \mathrm{~B}^{[48]}$ & VSMC proliferation; modulates neointimal formation \\
\hline miR-30c & PDGFR $\beta^{[51]}$ & PASMC proliferation and apoptosis \\
\hline miR-322 & $\operatorname{BMPR} 1 \alpha, \operatorname{Smad} 5^{[52]}$ & PASMC proliferation and migration \\
\hline
\end{tabular}




\begin{tabular}{|lll|}
\hline miR-130 & CDKN1A $^{[53]}$ & PASMC proliferation \\
Lnc-Ang362 & miR-221, mi-222 $^{[68]}$ & VSMC proliferation \\
LincRNA-p21 & MDM2 $^{\left[77^{* *}\right]}$ & VSMC proliferation and apoptosis; modulates neointimal formation \\
SMILR & HAS2 $^{[117]}$ & VSMC proliferation \\
& & \\
& \\
& \\
\end{tabular}

BMPR2 indicates bone morphogenetic protein receptor 2; PTEN, phosphatase and tensin homologue; BCL2, B-cell lymphoma 2; PDCD4, programmed cell death 4; TPM1, tropomyosin 1 (Alpha); SP1, specificity protein 1; DOCK4,5,7, dedicator of cytokinesis 4,5,7; STAT3, signal transducer and activator of transcription 3; STAB1, stabilin-1; YOD1, YOD1 deubiquitinase; CDKN1B, cyclin-dependent kinase inhibitor 1B; PDGFR $\beta$, beta-type platelet-derived growth factor receptor; BMPR1 $\alpha$, bone morphogenetic protein receptor type $1 \alpha$; CDKN1A, cyclin-dependent kinase inhibitor 1A; MDM2, E3 ubiquitin-protein ligase Mdm2; and HAS2, hyaluronan synthase 2.

\section{References and recommended reading}

* of special interest

** of outstanding interest

[1] Shimoda LA, Laurie SS. Vascular remodeling in pulmonary hypertension. 
Journal of molecular medicine 2013; 91:297-309.

[2] Pasterkamp G, de Kleijn DP, Borst C. Arterial remodeling in atherosclerosis, restenosis and after alteration of blood flow: potential mechanisms and clinical implications. Cardiovascular research 2000; 45:843-852.

[3] Gibbons GH, Dzau VJ. The emerging concept of vascular remodeling. The New England journal of medicine 1994; 330:1431-1438.

[4] Ponticos M, Smith BD. Extracellular matrix synthesis in vascular disease: hypertension, and atherosclerosis. Journal of biomedical research 2014; 28:25-39.

[5] Kipshidze N, Dangas G, Tsapenko M et al. Role of the endothelium in modulating neointimal formation: vasculoprotective approaches to attenuate restenosis after percutaneous coronary interventions. Journal of the American College of Cardiology 2004; 44:733-739.

[6] Lao KH, Zeng L, Xu Q. Endothelial and smooth muscle cell transformation in atherosclerosis. Current opinion in lipidology 2015; 26:449-456.

[7] Aihara K, Mogi M, Shibata R et al. Inflammation and vascular remodeling. International journal of vascular medicine 2012; 2012:596796.

[8] Otsuka F, Finn AV, Yazdani SK et al. The importance of the endothelium in atherothrombosis and coronary stenting. Nature reviews. Cardiology 2012; 9:439-453.

[9] Sprague AH, Khalil RA. Inflammatory cytokines in vascular dysfunction and vascular disease. Biochem Pharmacol 2009; 78:539-552. 
[10] Hassoun PM, Mouthon L, Barbera JA et al. Inflammation, growth factors, and pulmonary vascular remodeling. Journal of the American College of Cardiology 2009; 54:S10-19.

[11] Song X, Shan D, Chen J, Jing Q. miRNAs and IncRNAs in vascular injury and remodeling. Science China. Life sciences 2014; 57:826-835.

[12] Gareri C, De Rosa S, Indolfi C. MicroRNAs for Restenosis and Thrombosis After Vascular Injury. Circ Res 2016; 118:1170-1184.

[13] Leopold JA, Maron BA. Molecular Mechanisms of Pulmonary Vascular Remodeling in Pulmonary Arterial Hypertension. International journal of molecular sciences 2016; 17.

[14] Consortium EP. An integrated encyclopedia of DNA elements in the human genome. Nature 2012; 489:57-74.

[15] Samanta S, Balasubramanian S, Rajasingh S et al. MicroRNA: A new therapeutic strategy for cardiovascular diseases. Trends in cardiovascular medicine 2016 .

[16] Welten SM, Goossens EA, Quax PH, Nossent AY. The multifactorial nature of microRNAs in vascular remodelling. Cardiovascular research 2016; 110:6-22

[17] Ha M, Kim VN. Regulation of microRNA biogenesis. Nature reviews. Molecular cell biology 2014; 15:509-524.

[18] Krol J, Loedige I, Filipowicz W. The widespread regulation of microRNA biogenesis, function and decay. Nature reviews. Genetics 2010; 11:597-610. 
[19] Winter J, Jung S, Keller $S$ et al. Many roads to maturity: microRNA biogenesis pathways and their regulation. Nature cell biology 2009; 11:228-234.

[20] Romaine SP, Tomaszewski M, Condorelli G, Samani NJ. MicroRNAs in cardiovascular disease: an introduction for clinicians. Heart 2015; 101:921-928.

[21] Ono K, Kuwabara Y, Han J. MicroRNAs and cardiovascular diseases. The FEBS journal 2011; 278:1619-1633.

[22] Pasquinelli AE. MicroRNAs and their targets: recognition, regulation and an emerging reciprocal relationship. Nature reviews. Genetics 2012; 13:271-282.

[23] Maegdefessel L, Rayner KJ, Leeper NJ. MicroRNA regulation of vascular smooth muscle function and phenotype: early career committee contribution. Arteriosclerosis, thrombosis, and vascular biology 2015; 35:2-6.

[24] Watts JK, Corey DR. Silencing disease genes in the laboratory and the clinic. J Pathol 2012; 226:365-379.

[25] Zhu LH, Huang L, Zhang $X$ et al. Mindin regulates vascular smooth muscle cell phenotype and prevents neointima formation. Clinical science 2015; 129:129-145.

[26] George SJ, Wan S, Hu J et al. Sustained reduction of vein graft neointima formation by ex vivo TIMP-3 gene therapy. Circulation 2011; 124:S135-142. [27] Yin RX, Yang DZ, Wu JZ. Nanoparticle drug- and gene-eluting stents for 
the prevention and treatment of coronary restenosis. Theranostics 2014; $4: 175-200$.

[28] Jonigk D, Golpon H, Bockmeyer CL et al. Plexiform lesions in pulmonary arterial hypertension composition, architecture, and microenvironment. Am J Pathol 2011; 179:167-179.

[29] Ji R, Cheng $\mathrm{Y}$, Yue J et al. MicroRNA expression signature and antisense-mediated depletion reveal an essential role of MicroRNA in vascular neointimal lesion formation. Circ Res 2007; 100:1579-1588.

[30] Lin $Y$, Liu $X$, Cheng $Y$ et al. Involvement of MicroRNAs in hydrogen peroxide-mediated gene regulation and cellular injury response in vascular smooth muscle cells. J Biol Chem 2009; 284:7903-7913.

[31] Wang M, Li W, Chang GQ et al. MicroRNA-21 regulates vascular smooth muscle cell function via targeting tropomyosin 1 in arteriosclerosis obliterans of lower extremities. Arteriosclerosis, thrombosis, and vascular biology 2011; 31:2044-2053.

[32] Yang G, Pei Y, Cao Q, Wang R. MicroRNA-21 represses human cystathionine gamma-lyase expression by targeting at specificity protein-1 in smooth muscle cells. J Cell Physiol 2012; 227:3192-3200.

[33] Davis BN, Hilyard AC, Lagna G, Hata A. SMAD proteins control DROSHA-mediated microRNA maturation. Nature 2008; 454:56-61.

[34] Davis-Dusenbery BN, Chan MC, Reno KE et al. down-regulation of Kruppel-like factor-4 (KLF4) by microRNA-143/145 is critical for modulation of 
vascular smooth muscle cell phenotype by transforming growth factor-beta and bone morphogenetic protein 4. J Biol Chem 2011; 286:28097-28110. [35] Kang $\mathrm{H}$, Davis-Dusenbery BN, Nguyen $\mathrm{PH}$ et al. Bone morphogenetic protein 4 promotes vascular smooth muscle contractility by activating microRNA-21 (miR-21), which down-regulates expression of family of dedicator of cytokinesis (DOCK) proteins. J Biol Chem 2012; 287:3976-3986. [36] McDonald RA, White KM, Wu J et al. miRNA-21 is dysregulated in response to vein grafting in multiple models and genetic ablation in mice attenuates neointima formation. European heart journal 2013; 34:1636-1643. [37]** McDonald RA, Halliday CA, Miller AM et al. Reducing In-Stent Restenosis: Therapeutic Manipulation of miRNA in Vascular Remodeling and Inflammation. Journal of the American College of Cardiology 2015; 65:2314-2327.

This study demonstrated that genetic ablation of miR-21 attenuated neointimal formation and macrophage activation in an in-stent restenosis mouse model. The mechanism is via enhanced levels of anti-inflammatory M2 macrophages coupled with an impaired smooth muscle cell proliferation and migration.

[38] ${ }^{\star \star}$ Wang $\mathrm{D}$, Deuse $\mathrm{T}$, Stubbendorff $\mathrm{M}$ et al. Local MicroRNA Modulation Using a Novel Anti-miR-21-Eluting Stent Effectively Prevents Experimental In-Stent Restenosis. Arteriosclerosis, thrombosis, and vascular biology 2015; 35:1945-1953.

MiR-21 expression increased in human tissue samples in patients with ISR. 
Knocking down miR-21 dose-dependently reduced luminal obliteration in a humanized model. In addition, anti-miR-21-coated stents effectively reduced ISR compared with bare-metal stents, which demonstrated emerging technology for drug eluting stent applications.

[39] He M, Gong $\mathrm{Y}$, Shi $\mathrm{J}$ et al. Plasma microRNAs as potential noninvasive biomarkers for in-stent restenosis. PloS one 2014; 9:e112043.

[40] Yang S, Banerjee S, Freitas A et al. miR-21 regulates chronic hypoxia-induced pulmonary vascular remodeling. Am J Physiol Lung Cell Mol Physiol 2012; 302:L521-529.

[41] ${ }^{*}$ White K, Dempsie Y, Caruso P et al. Endothelial apoptosis in pulmonary hypertension is controlled by a microRNA/programmed cell death $4 /$ caspase- 3 axis. Hypertension 2014; 64:185-194.

This study use miR-21 genetic ablation (miR-21/-) and overexpression mice (CAG-miR-21 mice) demonstrated miR-21 prevented the development of PH in response to chromic hypoxia plus SU5416 injury. The miR-21-respoisive PDCD4/caspase-3 pathway induced endothelial apoptosis contributed to the protective phenotype of miR-21.

[42] Parikh VN, Jin RC, Rabello S et al. MicroRNA-21 integrates pathogenic signaling to control pulmonary hypertension: results of a network bioinformatics approach. Circulation 2012; 125:1520-1532.

[43] Bienertova-Vasku J, Novak J, Vasku A. MicroRNAs in pulmonary arterial hypertension: pathogenesis, diagnosis and treatment. Journal of the American 
Society of Hypertension : JASH 2015; 9:221-234.

[44] Gomez IG, MacKenna DA, Johnson BG et al. Anti-microRNA-21 oligonucleotides prevent Alport nephropathy progression by stimulating metabolic pathways. The Journal of clinical investigation 2015; 125:141-156.

[45] Wang $\mathrm{H}$, Jiang $M$, Xu Z et al. miR-146b-5p promotes VSMC proliferation and migration. International journal of clinical and experimental pathology 2015; 8:12901-12907.

[46] Kurakula K, Goumans MJ, Ten Dijke P. Regulatory RNAs controlling vascular (dys)function by affecting TGF-ss family signalling. EXCLI journal $2015 ; 14: 832-850$.

[47] Cao $\mathrm{H}, \mathrm{Hu} X$, Zhang $\mathrm{Q}$ et al. Upregulation of let-7a inhibits vascular smooth muscle cell proliferation in vitro and in vein graft intimal hyperplasia in rats. The Journal of surgical research 2014; 192:223-233.

[48] Wang XW, He XJ, Lee KC et al. MicroRNA-221 sponge therapy attenuates neointimal hyperplasia and improves blood flows in vein grafts. International journal of cardiology 2016; 208:79-86.

[49] Ohnaka M, Marui A, Yamahara K et al. Effect of microRNA-145 to prevent vein graft disease in rabbits by regulation of smooth muscle cell phenotype. The Journal of thoracic and cardiovascular surgery 2014; 148:676-682 e672. [50] ${ }^{* *}$ Deng L, Blanco FJ, Stevens $\mathrm{H}$ et al. MicroRNA-143 Activation Regulates Smooth Muscle and Endothelial Cell Crosstalk in Pulmonary Arterial Hypertension. Circulation research 2015; 117:870-883. 
This is a description of exosomal miR-143 mediated cell-to-cell communication between PASMCs and PAECs affecting cell migration and angiogenesis. MiR-143 inhibition prevented the development of $\mathrm{PH}$ in response to chronic hypoxia.

[51] Xing Y, Zheng X, Li G et al. MicroRNA-30c contributes to the development of hypoxia pulmonary hypertension by inhibiting platelet-derived growth factor receptor beta expression. The international journal of biochemistry \& cell biology 2015; 64:155-166.

[52] Zeng $\mathrm{Y}$, Liu $\mathrm{H}$, Kang $\mathrm{K}$ et al. Hypoxia inducible factor-1 mediates expression of miR-322: potential role in proliferation and migration of pulmonary arterial smooth muscle cells. Scientific reports 2015; 5:12098.

[53] Brock M, Haider TJ, Vogel J et al. The hypoxia-induced microRNA-130a controls pulmonary smooth muscle cell proliferation by directly targeting CDKN1A. The international journal of biochemistry \& cell biology 2015; 61:129-137.

[54] Sun M, Kraus WL. From discovery to function: the expanding roles of long noncoding RNAs in physiology and disease. Endocrine reviews 2015; $36: 25-64$.

[55] Quinn JJ, Chang HY. Unique features of long non-coding RNA biogenesis and function. Nature reviews. Genetics 2016; 17:47-62.

[56] Cabili MN, Trapnell C, Goff L et al. Integrative annotation of human large intergenic noncoding RNAs reveals global properties and specific subclasses. 
Genes \& development 2011; 25:1915-1927.

[57] Mercer TR, Dinger ME, Mattick JS. Long non-coding RNAs: insights into functions. Nature reviews. Genetics 2009; 10:155-159.

[58] Grote P, Wittler L, Hendrix D et al. The tissue-specific IncRNA Fendrr is an essential regulator of heart and body wall development in the mouse. Developmental cell 2013; 24:206-214.

[59] Thum T, Condorelli G. Long noncoding RNAs and microRNAs in cardiovascular pathophysiology. Circulation research 2015; 116:751-762.

[60] Rinn JL, Chang HY. Genome regulation by long noncoding RNAs. Annual review of biochemistry 2012; 81:145-166.

[61] Wang KC, Chang HY. Molecular mechanisms of long noncoding RNAs. Molecular cell 2011; 43:904-914.

[62] Guttman M, Rinn JL. Modular regulatory principles of large non-coding RNAs. Nature 2012; 482:339-346.

[63] Hu W, Alvarez-Dominguez JR, Lodish HF. Regulation of mammalian cell differentiation by long non-coding RNAs. EMBO reports 2012; 13:971-983.

[64] Congrains A, Kamide K, Oguro R et al. Genetic variants at the 9p21 locus contribute to atherosclerosis through modulation of ANRIL and CDKN2A/B. Atherosclerosis 2012; 220:449-455.

[65] Motterle A, Pu X, Wood $\mathrm{H}$ et al. Functional analyses of coronary artery disease associated variation on chromosome 9p21 in vascular smooth muscle cells. Human molecular genetics 2012; 21:4021-4029. 
[66] Congrains A, Kamide K, Katsuya $T$ et al. CVD-associated non-coding RNA, ANRIL, modulates expression of atherogenic pathways in VSMC. Biochemical and biophysical research communications 2012; 419:612-616.

[67] Zhou X, Han X, Wittfeldt A et al. Long non-coding RNA ANRIL regulates inflammatory responses as a novel component of NF-kappaB pathway. RNA biology 2016; 13:98-108.

[68] Leung A, Trac C, Jin W et al. Novel long noncoding RNAs are regulated by angiotensin II in vascular smooth muscle cells. Circulation research 2013; 113:266-278.

[69] Chistiakov DA, Sobenin IA, Orekhov AN, Bobryshev YV. Human miR-221/222 in Physiological and Atherosclerotic Vascular Remodeling. BioMed research international 2015; 2015:354517.

[70] Liu X, Cheng Y, Zhang S et al. A necessary role of miR-221 and miR-222 in vascular smooth muscle cell proliferation and neointimal hyperplasia. Circulation research 2009; 104:476-487.

[71] ${ }^{*}$ Bell $\mathrm{RD}$, Long $\mathrm{X}$, Lin $\mathrm{M}$ et al. Identification and initial functional characterization of a human vascular cell-enriched long noncoding RNA. Arteriosclerosis, thrombosis, and vascular biology 2014; 34:1249-1259.

First RNA-Seq study in human coronary artery SMC for discovery of IncRNAs, which identified a novel vascular cell-enriched, cytoplasmic IncRNA SENCR. The functions of SENCR include stabilization the smooth muscle cell contractile phenotype and cell migration. 
[72] Boulberdaa M, Scott E, Ballantyne M et al. A Role for the Long Noncoding RNA SENCR in Commitment and Function of Endothelial Cells. Molecular therapy : the journal of the American Society of Gene Therapy 2016.

$[73]^{* *} \mathrm{Wu}$ G, Cai J, Han Y et al. LincRNA-p21 regulates neointima formation, vascular smooth muscle cell proliferation, apoptosis, and atherosclerosis by enhancing p53 activity. Circulation 2014; 130:1452-1465.

Study showed lincRNA-p21 expression is decreased in patients with coronary artery disease and atherosclerotic plaques of $\mathrm{ApoE}^{-/}$mice. In vitro experiments demonstrated lincRNA-p21 repressed cell proliferation and induced apoptosis in VSMC and mononuclear macrophage cells. In vivo carotid artery injury model revealed that inhibition of lincRNA-p21 resulted in neointimal hyperplasia. Mechanistically, this showed that lincRNA-p21 is a target of p53 and feedback on the function of p53 via binding to MDM2.

[74] He C, Ding JW, Li S et al. The Role of Long Intergenic Noncoding RNA p21 in Vascular Endothelial Cells. DNA and cell biology 2015; 34:677-683.

[75] Tang SS, Zheng BY, Xiong XD. LincRNA-p21: Implications in Human Diseases. International journal of molecular sciences 2015; 16:18732-18740. $[76]^{\star *}$ Ballantyne MD, Pinel K, Dakin R et al. Smooth Muscle Enriched Long Non-Coding RNA (SMILR) Regulates Cell Proliferation. Circulation 2016. RNA-Seq performed on human saphenous vein (HSV) VSMCs following stimulation with IL-1 $1 \alpha$ and PDGF revealed a novel IncRNA SMILR, which reduced cell proliferation and HAS2 gene expression following knockdown by 
SiRNA. In unstable atherosclerotic plaques and plasma from patients with high plasma C-reactive protein, elevated levels of this IncRNA were observed associating dysregulation with human pathology.

[77] Liu JY, Yao J, Li XM et al. Pathogenic role of IncRNA-MALAT1 in endothelial cell dysfunction in diabetes mellitus. Cell death \& disease 2014; 5:e1506.

$[78]^{* *}$ Michalik KM, You X, Manavski $Y$ et al. Long noncoding RNA MALAT1 regulates endothelial cell function and vessel growth. Circulation research $2014 ; 114: 1389-1397$.

Hypoxia responsive IncRNA MALAT1 silencing induced a promigratiory response and increased basal sprouting and migration, while inhibited endothelial proliferation. Genetic ablation and pharmacological of MALAT1 inhibited proliferation of ECs, reduced neonatal retina vascularization and blood flow recovery and capillary density in hind limb ischemia mice model. This result revealed the important role of MALAT1 in the balance of proliferative and migratory endothelial cell phenotypes in vitro.

[79] Voellenkle C, Garcia-Manteiga JM, Pedrotti S et al. Implication of Long noncoding RNAs in the endothelial cell response to hypoxia revealed by RNA-sequencing. Scientific reports 2016; 6:24141.

[80] Boon RA, Jae N, Holdt L, Dimmeler S. Long Noncoding RNAs: From Clinical Genetics to Therapeutic Targets? Journal of the American College of Cardiology 2016; 67:1214-1226. 
[81] Lorenzen JM, Thum T. Long noncoding RNAs in kidney and cardiovascular diseases. Nature reviews. Nephrology 2016.

[82] Uchida S, Dimmeler S. Long noncoding RNAs in cardiovascular diseases. Circulation research 2015; 116:737-750.

[83] McCoy-Simandle K, Hanna SJ, Cox D. Exosomes and nanotubes: Control of immune cell communication. The international journal of biochemistry \& cell biology 2016; 71:44-54.

[84] Ciardiello C, Cavallini L, Spinelli C et al. Focus on Extracellular Vesicles: New Frontiers of Cell-to-Cell Communication in Cancer. International journal of molecular sciences 2016; 17.

[85] Thery C, Ostrowski M, Segura E. Membrane vesicles as conveyors of immune responses. Nat Rev Immunol 2009; 9:581-593.

[86] Bang C, Thum T. Exosomes: new players in cell-cell communication. The international journal of biochemistry \& cell biology 2012; 44:2060-2064.

[87] Valadi $\mathrm{H}$, Ekstrom K, Bossios $\mathrm{A}$ et al. Exosome-mediated transfer of mRNAs and microRNAs is a novel mechanism of genetic exchange between cells. Nature cell biology 2007; 9:654-659.

[88] Kosaka N. Decoding the Secret of Cancer by Means of Extracellular Vesicles. Journal of clinical medicine 2016; 5.

[89] Waldenstrom A, Ronquist G. Role of exosomes in myocardial remodeling. Circulation research 2014; 114:315-324.

[90] Yellon DM, Davidson SM. Exosomes: nanoparticles involved in 
cardioprotection? Circulation research 2014; 114:325-332.

[91] Sahoo S, Losordo DW. Exosomes and cardiac repair after myocardial infarction. Circulation research 2014; 114:333-344.

[92] Loyer X, Vion AC, Tedgui A, Boulanger CM. Microvesicles as cell-cell messengers in cardiovascular diseases. Circulation research 2014; 114:345-353.

[93] Kishore R, Khan M. More Than Tiny Sacks: Stem Cell Exosomes as Cell-Free Modality for Cardiac Repair. Circulation research 2016; 118:330-343.

[94] Melo SA, Sugimoto H, O'Connell JT et al. Cancer exosomes perform cell-independent microRNA biogenesis and promote tumorigenesis. Cancer cell 2014; 26:707-721.

[95] Hergenreider E, Heydt S, Treguer $\mathrm{K}$ et al. Atheroprotective communication between endothelial cells and smooth muscle cells through miRNAs. Nature cell biology $2012 ; 14: 249-256$.

[96] $^{* *}$ Climent $M$, Quintavalle $M$, Miragoli $M$ et al. TGFbeta Triggers miR-143/145 Transfer From Smooth Muscle Cells to Endothelial Cells, Thereby Modulating Vessel Stabilization. Circulation research 2015; 116:1753-1764.

MiR-143/145 act as communication molecules mediated cell to cell communication between SMCs and ECs via tunnelling nanotubes, which is modulated by the TGF- $\beta$ pathway. In ECs, miR-143/145 target HKII and 
(ITGß8)-2 respectively to modulate angiogenesis and proliferation.

[97] Zhou J, Li YS, Nguyen P et al. Regulation of vascular smooth muscle cell turnover by endothelial cell-secreted microRNA-126: role of shear stress. Circulation research 2013; 113:40-51.

$[98]^{*}$ Lin X, He Y, Hou X et al. Endothelial Cells Can Regulate Smooth Muscle Cells in Contractile Phenotype through the miR-206/ARF6\&NCX1/Exosome Axis. PloS one 2016; 11:e0152959.

MiR-206 targets ARF6 and NCX1 to function as a regulator of exosome production in HUVECs, which further affects the secretion and transfer of exosomal miR-26 from HUVECs to VSMCs.

[99] van Balkom BW, de Jong OG, Smits $M$ et al. Endothelial cells require miR-214 to secrete exosomes that suppress senescence and induce angiogenesis in human and mouse endothelial cells. Blood 2013; 121:3997-4006, S3991-3915.

[100] Botti G, Marra L, Malzone MG et al. LncRNA HOTAIR as prognostic circulating marker and potential therapeutic target in patients with tumor diseases. Current drug targets 2015.

[101] Conigliaro A, Costa V, Lo Dico A et al. CD90+ liver cancer cells modulate endothelial cell phenotype through the release of exosomes containing $\mathrm{H} 19$ IncRNA. Molecular cancer 2015; 14:155.

[102] Takahashi K, Yan IK, Kogure T et al. Extracellular vesicle-mediated transfer of long non-coding RNA ROR modulates chemosensitivity in human 
hepatocellular cancer. FEBS open bio 2014; 4:458-467.

[103] Takahashi K, Yan IK, Wood J et al. Involvement of extracellular vesicle long noncoding RNA (linc-VLDLR) in tumor cell responses to chemotherapy. Molecular cancer research : MCR 2014; 12:1377-1387.

[104] Kogure T, Yan IK, Lin WL, Patel T. Extracellular Vesicle-Mediated Transfer of a Novel Long Noncoding RNA TUC339: A Mechanism of Intercellular Signaling in Human Hepatocellular Cancer. Genes \& cancer 2013; $4: 261-272$.

[105] Isin M, Uysaler E, Ozgur E et al. Exosomal IncRNA-p21 levels may help to distinguish prostate cancer from benign disease. Frontiers in genetics 2015; 6:168.

[106] Gezer U, Ozgur E, Cetinkaya M et al. Long non-coding RNAs with low expression levels in cells are enriched in secreted exosomes. Cell biology international 2014; 38:1076-1079.

[107] Sanchez-Nino MD, Ortiz A. HCV infection and miravirsen. The New England journal of medicine 2013; 369:877-878.

[108] Janssen HL, Reesink HW, Lawitz EJ et al. Treatment of HCV infection by targeting microRNA. The New England journal of medicine 2013; 368:1685-1694.

[109] Adams BD, Parsons C, Slack FJ. The tumor-suppressive and potential therapeutic functions of miR-34a in epithelial carcinomas. Expert opinion on therapeutic targets 2015:1-17. 
[110] Trajkovski M, Hausser J, Soutschek J et al. MicroRNAs 103 and 107 regulate insulin sensitivity. Nature 2011; 474:649-653.

[111] Li Z, Rana TM. Therapeutic targeting of microRNAs: current status and future challenges. Nature reviews. Drug discovery 2014; 13:622-638.

[112] Xiao J, Meng XM, Huang XR et al. miR-29 inhibits bleomycin-induced pulmonary fibrosis in mice. Molecular therapy : the journal of the American Society of Gene Therapy 2012; 20:1251-1260.

[113] Butovsky O, Jedrychowski MP, Cialic R et al. Targeting miR-155 restores abnormal microglia and attenuates disease in SOD1 mice. Annals of neurology 2015; 77:75-99.

$[114]^{*}$ van Rooij E, Kauppinen S. Development of microRNA therapeutics is coming of age. EMBO molecular medicine 2014; 6:851-864.

A comprehensive review describing the current strategies for therapeutic modulation of miRNAs.

[115] Ballantyne MD, McDonald RA, Baker AH. IncRNA/MicroRNA interactions in the vasculature. Clinical pharmacology and therapeutics 2016; 99:494-501. [116] Djebali S, Davis CA, Merkel A et al. Landscape of transcription in human cells. Nature 2012; 489:101-108.

[117] Ballantyne MD, Pinel K, Dakin R et al. Smooth Muscle Enriched Long Noncoding RNA (SMILR) Regulates Cell Proliferation. Circulation 2016; 133:2050-2065. 


\section{Figure Legends}

Figure 1: Overview of the genome location and functions of lncRNAs

(A). Based on their genome locations, lncRNAs can be classified into Sense: transcribed from the sense strand of a protein-coding gene; Antisense: transcribed from the antisense strand of a protein-coding gene; Intronic: transcribed entirely from an intron of a protein-coding gene; Intergenic: transcribed sequences is not located near any other protein-coding loci; Bidirectional: transcribed sequences are located on the opposite strand from a protein-coding gene whose transcription is initiated less than 1000 base pairs away. (B). The main functions of lncRNA including SIGNALLING: IncRNA can function as molecular signal or indicator of transcriptional activity; DECOY: IncRNAs can preclude the access of regulatory proteins to DNA; GUIDES: lncRNA are be required for the localisation of specific protein complexes either in cis (on neighbouring genes) or in trans (on distantly located genes); SCAFFOLDS: lncRNAs can serve as adaptors to bring two or more proteins into discrete complexes.

Figure 2: Cell-to-cell communication between vascular smooth muscle and endothelial cells through miRNAs

Working models were showed to illustrate cell-to-cell communication between VSMCs and ECs. (A). Various stimuli induce exosomal miR-143 secretion in PASMCs, which can be taken up by EC to induce cell migration and angiogenesis [50**]. (B). Shear stress can induce KLF2 and miR-143/145 expression and inhibit 
miR-126 expression in EC. MiR-143/145 and miR-126 transfer to VSMCs through exosomes, and Ago2-facilitated miRNA transmission affects target genes and cellular functions in VSMCs, leading to atheroprotective phenotype [95, 97]. (C). TGF- $\beta$ induced miR-143 expression in VSMCs, which can be transferred to ECs through TNTs to inhibit target gene expression and induce cell proliferation and angiogenesis [96]. (D). MiR-206 binding to 3'UTR of ARF6/NCX1 in EC inhibits exosomal miR-26a secretion and uptake into VSMCs, where they regulate the SMC contractile phenotype [98]. 
Table 1 Overview of confirmed target genes of ncRNAs in VSMCs

\begin{tabular}{|c|c|c|}
\hline ncRNA & Target genes & Effect on cell behaviour/neointimal formation \\
\hline \multirow[t]{8}{*}{$\operatorname{miR}-21$} & $\mathrm{PTEN}^{\left[29,38^{* *}\right]}, \mathrm{BCL}^{[29]}$ & VSMC proliferation and apoptosis \\
\hline & $\operatorname{PDCD} 4^{[30,37 * *]}$ & VSMC apoptosis, proliferation, and migration \\
\hline & $\mathrm{TPM}^{[31]}$ & VSMC proliferation and migration \\
\hline & $\mathrm{SP} 1^{[32]}$ & VSMC proliferation and differentiation \\
\hline & DOCK $4,5,7^{[35]}$ & VSMC migration and contractility \\
\hline & STAT3 $^{\left[36,3^{* *}\right]}, \mathrm{PTEN}$, & \\
\hline & $\mathrm{BMPR}^{[36]}$ & VSMC proliferation, migration; modulates neointimal formation \\
\hline & BMPR2, STAB1, YOD1 ${ }^{[40]}$ & PASMC proliferation and apoptosis \\
\hline miR-221 & $\mathrm{CDKN}^{\left[\mathrm{B}^{[48]}\right.}$ & VSMC proliferation; modulates neointimal formation \\
\hline miR-30c & PDGFR $\beta^{[51]}$ & PASMC proliferation and apoptosis \\
\hline miR-322 & $\operatorname{BMPR} 1 \alpha, \operatorname{Smad} 5^{[52]}$ & PASMC proliferation and migration \\
\hline miR-130 & CDKN1A ${ }^{[53]}$ & PASMC proliferation \\
\hline Lnc-Ang362 & $\operatorname{miR}-221, \mathrm{mi}-222^{[68]}$ & VSMC proliferation \\
\hline LincRNA-p21 & $\operatorname{MDM} 2^{\left[73^{* *}\right]}$ & VSMC proliferation and apoptosis; modulates neointimal formation \\
\hline SMILR & $\mathrm{HAS2}^{[117]}$ & VSMC proliferation \\
\hline
\end{tabular}

BMPR2 indicates bone morphogenetic protein receptor 2; PTEN, phosphatase and tensin homologue;

BCL2, B-cell lymphoma 2; PDCD4, programmed cell death 4; TPM1, tropomyosin 1 (Alpha); SP1, specificity protein 1; DOCK4,5,7, dedicator of cytokinesis 4,5,7; STAT3, signal transducer and activator of transcription 3; STAB1, stabilin-1; YOD1, YOD1 deubiquitinase; CDKN1B, cyclin-dependent kinase inhibitor 1B; PDGFR $\beta$, beta-type platelet-derived growth factor receptor; BMPR1 $\alpha$, bone morphogenetic protein receptor type $1 \alpha$; CDKN1A, cyclin-dependent kinase inhibitor 1A; MDM2, E3 ubiquitin-protein ligase Mdm2; and HAS2, hyaluronan synthase 2. 

A

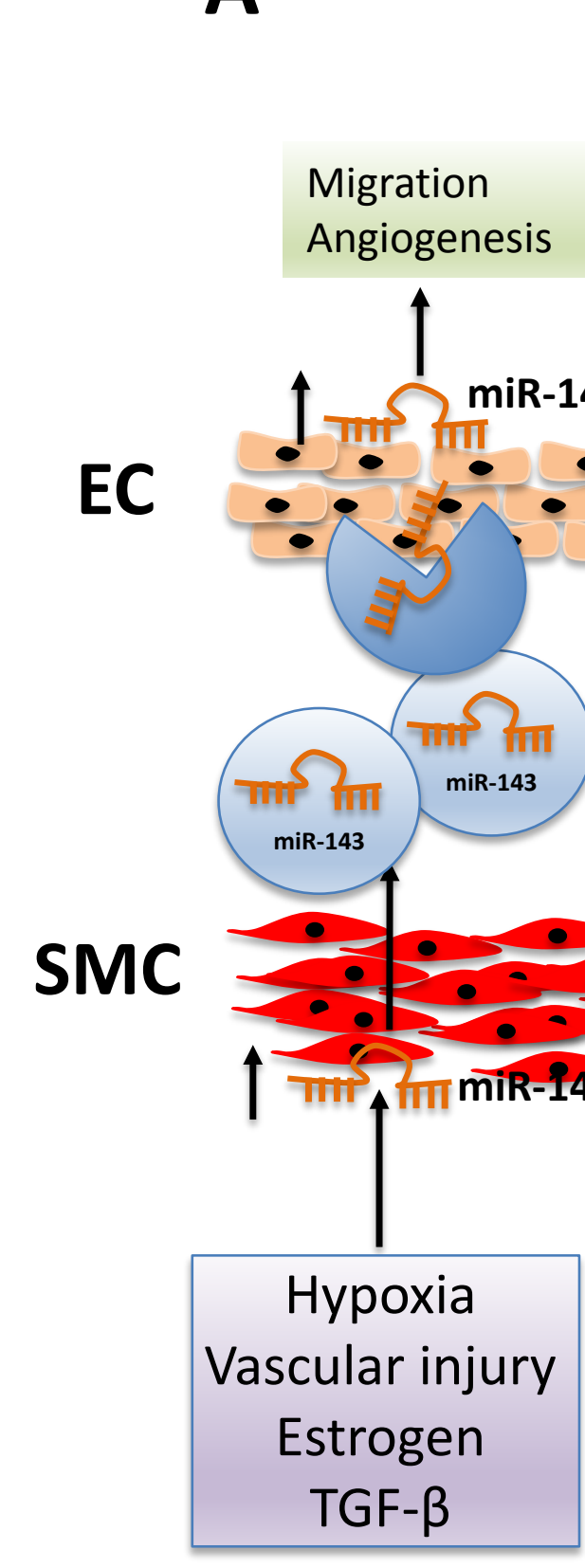

B

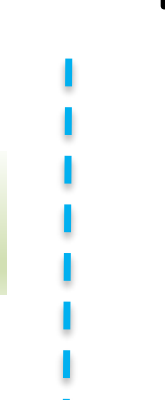

Shear stress

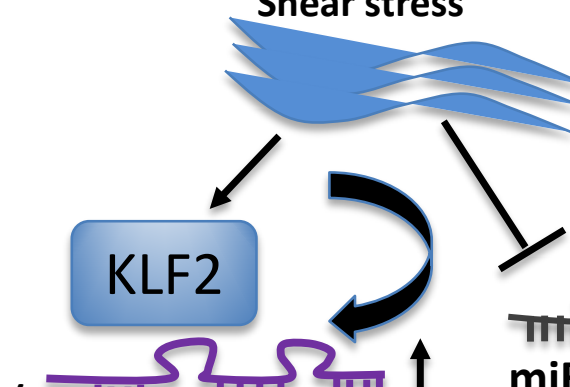

C

D
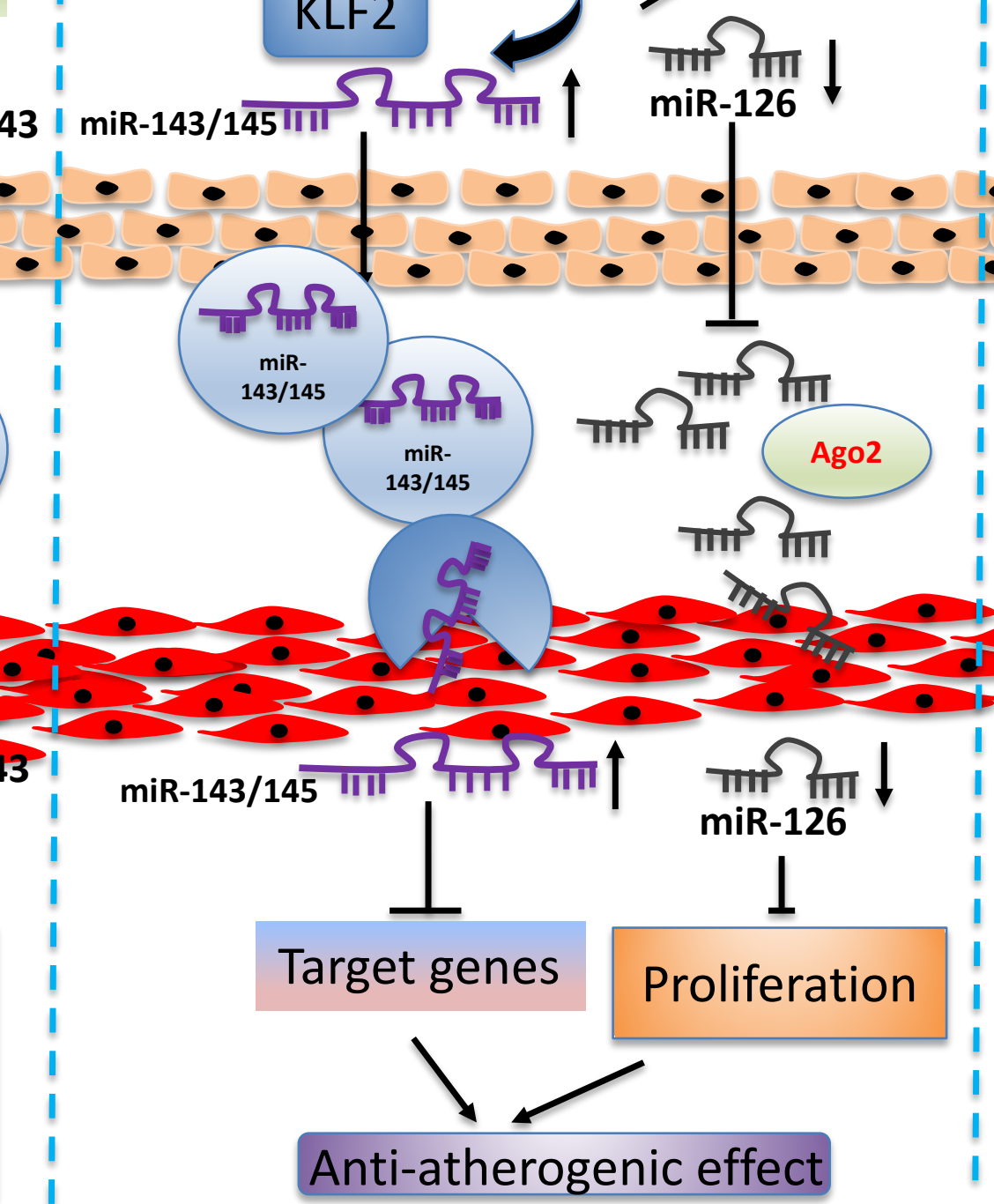

miR-206

Target genes Proliferation Angiogenesis

ARF6/NCX1
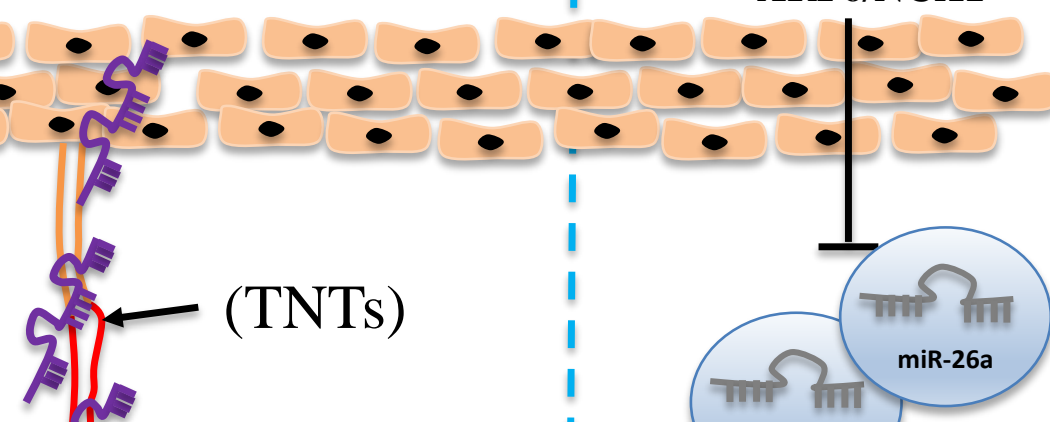

miR-143/145ात खाता काता।

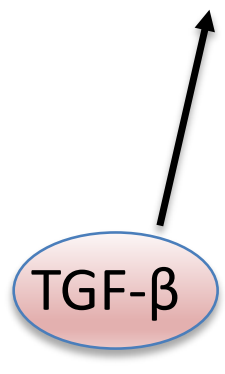

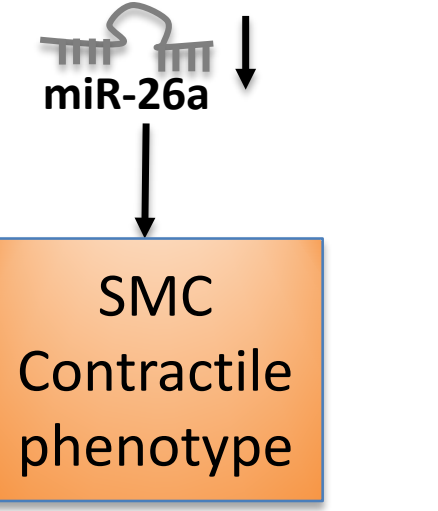

